\title{
UNITARY HECKE ALGEBRA MODULES WITH NONZERO DIRAC COHOMOLOGY
}

\author{
DAN BARBASCH AND DAN CIUBOTARU \\ To Nolan Wallach with admiration
}

\begin{abstract}
In this paper, we review the construction of the Dirac operator for graded affine Hecke algebras and calculate the Dirac cohomology of irreducible unitary modules for the graded Hecke algebra of $g l(n)$.
\end{abstract}

\section{INTRODUCTION}

The Dirac operator plays an important role in the representation theory of real reductive Lie groups. An account of the definition, properties and some applications can be found in $\mathrm{BW}$. It is well known, starting with the work of $\mathrm{AS}$ and $\mathrm{P}$, that discrete series occur in the kernel of the Dirac operator. Work of Enright and Wallach EW] generalizes these results to other types of representations. Other uses are to provide, via the Dirac inequality, introduced by Parthasarathy, necessary conditions for unitarity. One of the most striking applications is that for regular integral infinitesimal character, the Dirac inequality gives precisely the unitary dual, and determines the unitary representations with nontrivial $(\mathfrak{g}, K)$-cohomology.

Given these properties, Vogan has introduced the notion of Dirac cohomology; this was studied extensively in $[\mathrm{HP}$ and subsequent work. One can argue that Dirac cohomology is a generalization of $(\mathfrak{g}, K)$-cohomology. While a representation has nontrivial $(\mathfrak{g}, K)$-cohomology only if its infinitesimal character is regular integral, the corresponding condition necessary for Dirac cohomology to be nonzero is more general; certain representations with singular and nonintegral infinitesimal character will also have nontrivial Dirac cohomology.

In this paper, we prove new results about an analogue of the Dirac operator in the case of the graded affine Hecke algebra, introduced in $\overline{B C T}$. This operator can be thought of as the analogue of the Dirac operator in the case of a $p$-adic group. One of our results is to determine the behaviour of the Dirac cohomology with respect to Harish-Chandra type induction. In the real case, a unitary representation has nontrivial $(\mathfrak{g}, K)$-cohomology, if and only if it is (essentially) obtained from the trivial representation on a Levi component via the derived functor construction. For unitary representations with nontrivial Dirac cohomology the infinitesimal character can be nonintegral and singular. So conjecture instead, that unitary representations with nontrivial Dirac cohomology are all cohomologically induced from unipotent (in the sense of [A]) representations. To investigate this

The first author was partially supported by NSF grants DMS-0967386, DMS-0901104 and an NSA-AMS grant. The second author was partially supported by NSF DMS-0968065 and NSAAMS 081022. 
conjecture we explore the Dirac cohomology of unipotent representations for graded affine Hecke algebras. In particular, we compute part of the cohomology of spherical unipotent representations for affine Hecke algebras of all types. In the case of type $A$ we go further; we compute the cohomology of all unitary modules.

This paper was written while we were guests of the Max Planck Institute in Bonn as part of the program Analysis on Lie groups. We would like to thank the institute for its hospitality, and the organizers for making the program possible, and providing the environment to do this research.

\section{Dirac cohomology for graded Hecke algebras}

In this section we review the construction and properties of the Dirac operator from $\mathrm{BCT}$ and the classification of spin projective Weyl group representations from $\mathrm{Ci}$.

2.1. Root systems. We fix an $\mathbb{R}$-root system $\Phi=\left(V, R, V^{\vee}, R^{\vee}\right): V, V^{\vee}$ are finite dimensional $\mathbb{R}$-vector spaces, with a perfect bilinear pairing $():, V \times V^{\vee} \rightarrow \mathbb{R}$, so that $R \subset V \backslash\{0\}, R^{\vee} \subset V^{\vee} \backslash\{0\}$ are finite subsets in bijection

$$
R \longleftrightarrow R^{\vee}, \alpha \longleftrightarrow \alpha^{\vee} \text {, such that }\left(\alpha, \alpha^{\vee}\right)=2 .
$$

The reflections

$s_{\alpha}: V \rightarrow V, s_{\alpha}(v)=v-\left(v, \alpha^{\vee}\right) \alpha, \quad s_{\alpha}: V^{\vee} \rightarrow V^{\vee}, s_{\alpha}\left(v^{\prime}\right)=v^{\prime}-\left(\alpha, v^{\prime}\right) \alpha^{\vee}, \quad \alpha \in R$,

leave $R$ and $R^{\vee}$ invariant, respectively. Let $W$ be the subgroup of $G L(V)$ (respectively $\left.G L\left(V^{\vee}\right)\right)$ generated by $\left\{s_{\alpha}: \alpha \in R\right\}$.

We will assume that the root system $\Phi$ is reduced and crystallographic. We will fix a choice of simple roots $\Pi \subset R$, and consequently, positive roots $R^{+}$and positive coroots $R^{\vee,+}$. Often, we will write $\alpha>0$ or $\alpha<0$ in place of $\alpha \in R^{+}$or $\alpha \in\left(-R^{+}\right)$, respectively.

We fix a $W$-invariant inner product $\langle$,$\rangle on V$. Denote also by $\langle$,$\rangle the dual$ inner product on $V^{\vee}$. If $v$ is a vector in $V$ or $V^{\vee}$, we denote $|v|:=\langle v, v\rangle^{1 / 2}$.

2.2. The Clifford algebra. A classical reference for the Clifford algebra is Ch (see also section II.6 in $[\mathrm{BW}]$ ). Denote by $C(V)$ the Clifford algebra defined by $V$ and the inner product $\langle$,$\rangle . More precisely, C(V)$ is the quotient of the tensor algebra of $V$ by the ideal generated by

$$
\omega \otimes \omega^{\prime}+\omega^{\prime} \otimes \omega+2\left\langle\omega, \omega^{\prime}\right\rangle, \quad \omega, \omega^{\prime} \in V .
$$

Equivalently, $C(V)$ is the associative algebra with unit generated by $V$ with relations:

$$
\omega \omega^{\prime}+\omega^{\prime} \omega=-2\left\langle\omega, \omega^{\prime}\right\rangle .
$$

Let $\mathrm{O}(V)$ denote the group of orthogonal transformation of $V$ with respect to $\langle$,$\rangle .$ This acts by algebra automorphisms on $C(V)$, and the action of $-1 \in \mathrm{O}(V)$ induces a grading

$$
C(V)=C(V)_{\text {even }}+C(V)_{\text {odd }} .
$$

Let $\epsilon$ be the automorphism of $C(V)$ which is +1 on $C(V)_{\text {even }}$ and -1 on $C(V)_{\text {odd }}$. Let ${ }^{t}$ be the transpose antiautomorphism of $C(V)$ characterized by

$$
\omega^{t}=-\omega, \omega \in V, \quad(a b)^{t}=b^{t} a^{t}, a, b \in C(V) .
$$


The Pin group is

$$
\operatorname{Pin}(V)=\left\{a \in C(V): \epsilon(a) V a^{-1} \subset V, a^{t}=a^{-1}\right\} .
$$

It sits in a short exact sequence

$$
1 \longrightarrow \mathbb{Z} / 2 \mathbb{Z} \longrightarrow \operatorname{Pin}(V) \stackrel{p}{\longrightarrow} \mathrm{O}(V) \longrightarrow 1
$$

where the projection $p$ is given by $p(a)(\omega)=\epsilon(a) \omega a^{-1}$.

If $\operatorname{dim} V$ is even, the Clifford algebra $C(V)$ has a unique (up to equivalence) complex simple module $(\gamma, S)$ of dimension $2^{\operatorname{dim} V / 2}$, endowed with a positive definite Hermitian form $\langle,\rangle_{S}$ such that

$$
\left\langle\gamma(a) s, s^{\prime}\right\rangle_{S}=\left\langle s, \gamma\left(a^{t}\right) s^{\prime}\right\rangle_{S}, \quad \text { for all } a \in C(V) \text { and } s, s^{\prime} \in S .
$$

When $\operatorname{dim} V$ is odd, there are two simple inequivalent complex modules $\left(\gamma_{+}, S^{+}\right)$, $\left(\gamma_{-}, S^{-}\right)$of dimension $2^{[\operatorname{dim} V / 2]}$. Analogous to (2.2.6), these modules admit an invariant positive definite Hermitian form. In order to simplify the formulation of the results, we will often refer to any one of $S, S^{+}, S^{-}$, as a spin module.

Via (2.2.4), a spin module $S$ is an irreducible unitary $\operatorname{Pin}(V)$ representation.

2.3. The pin cover $\widetilde{W}$ of the Weyl group. The Weyl group $W$ acts by orthogonal transformations on $V$, so one can embed $W$ as a subgroup of $\mathrm{O}(V)$. We define the group $\widetilde{W}$ in $\operatorname{Pin}(V)$ :

$$
\widetilde{W}:=p^{-1}(W) \subset \operatorname{Pin}(V) \text {, where } p \text { is as in (2.2.5). }
$$

The group $\widetilde{W}$ has a Coxeter presentation similar to that of $W$. Recall that as a Coxeter group, $W$ has a presentation:

$$
W=\left\langle s_{\alpha}, \alpha \in \Pi \mid\left(s_{\alpha} s_{\beta}\right)^{m(\alpha, \beta)}=1, \alpha, \beta \in \Pi\right\rangle,
$$

for certain positive integers $m(\alpha, \beta)$. Theorem 3.2 in $[\mathrm{Mo}$ exhibits $\widetilde{W}$ as

$$
\widetilde{W}=\left\langle z, \widetilde{s}_{\alpha}, \alpha \in \Pi \mid z^{2}=1,\left(\widetilde{s}_{\alpha} \widetilde{s}_{\beta}\right)^{m(\alpha, \beta)}=z, \alpha, \beta \in \Pi\right\rangle .
$$

We call a representation $\widetilde{\sigma}$ of $\widetilde{W}$ genuine (resp. non-genuine) if $\widetilde{\sigma}(z)=-1$ (resp. $\widetilde{\sigma}(z)=1)$. The non-genuine $\widetilde{W}$-representations are the ones that factor through $W$. We say that two genuine $\widetilde{W}$-types $\sigma_{1}, \sigma_{2}$ are associate if $\sigma_{1} \cong \sigma_{2} \otimes$ sign.

Since $\widetilde{W} \subset \operatorname{Pin}(V)$, we can regard $S$ if $\operatorname{dim} V$ is even (resp. $S^{ \pm}$if $\operatorname{dim} V$ is odd) as unitary (genuine) $\widetilde{W}$-representations. If $R$ spans $V$, they are irreducible representations (피 Theorem 3.3]). When $\operatorname{dim} V$ is odd, $S^{+}$and $S^{-}$are associate, while if $\operatorname{dim} V$ is even, $S$ is self-associate.

Definition 2.3.1 ([BCT, §3.4]). Define the Casimir element of $\widetilde{W}$ :

$$
\Omega_{\widetilde{W}}=z \sum_{\substack{\alpha>0, \beta>0 \\ s_{\alpha}(\beta)<0}}\left|\alpha^{\vee} \| \beta^{\vee}\right| \widetilde{s}_{\alpha} \widetilde{s}_{\beta} \in \mathbb{C}[\widetilde{W}]^{\widetilde{W}} .
$$

Every $\widetilde{\sigma} \in \widehat{\widetilde{W}}$ acts on $\Omega_{\widetilde{W}}$ by a scalar, which we denote $\widetilde{\sigma}\left(\Omega_{\widetilde{W}}\right)$.

Before stating Theorem 2.3.1 we need to introduce more notation. Assume that $R$ spans $V$ and let $\mathfrak{g}$ be the complex semisimple Lie algebra with root system $\Phi$ and Cartan subalgebra $\mathfrak{h}=V^{\vee} \otimes_{\mathbb{R}} \mathbb{C}$, and let $G$ be the simply connected Lie group 
with Lie algebra $\mathfrak{g}$. Extend the inner product from $V^{\vee}$ to $\mathfrak{h}$. Let us denote by $\mathcal{T}(G)$ the set of $G$-conjugacy classes of Jacobson-Morozov triples $(e, h, f)$ in $\mathfrak{g}$. We set:

$\mathcal{T}_{0}(G)=\{[(e, h, f)] \in \mathcal{T}(G)$ : the centralizer of $\{e, h, f\}$ in $\mathfrak{g}$ is a toral subalgebra $\}$.

For every class in $\mathcal{T}(G)$, we may (and will) choose a representative $(e, h, f)$ such that $h \in \mathfrak{h}$. For every nilpotent element $e$, let $A(e)$ denote the A-group in $G$, and let $\widehat{A(e)})_{0}$ denote the set of representations of $A(e)$ of Springer type. For every $\phi \in \widehat{A(e)_{0}}$, let $\sigma_{(e, \phi)}$ be the associated Springer representation. Normalize the Springer correspondence so that $\sigma_{0, \text { triv }}=$ sign.

Theorem 2.3.1 ([i]). There is a surjective map

$$
\Psi: \widehat{\widetilde{W}}_{\text {gen }} \longrightarrow \mathcal{T}_{0}(G)
$$

with the following properties:

(1) If $\Psi(\widetilde{\sigma})=[(e, h, f)]$, then we have

$$
\widetilde{\sigma}\left(\Omega_{\widetilde{W}}\right)=\langle h, h\rangle,
$$

where $\Omega_{\widetilde{W}}$ is as in 2.3.4).

(2) Let $(e, h, f) \in \mathcal{T}_{0}(G)$ be given. For every Springer representation $\sigma_{(e, \phi)}$, $\phi \in \widehat{A(e)})_{0}$, and every spin $\widetilde{W}$-module $S$, there exists $\widetilde{\sigma} \in \Psi^{-1}[(e, h, f)]$ such that $\widetilde{\sigma}$ appears with nonzero multiplicity in the tensor product $\sigma_{(e, \phi)} \otimes S$. Conversely, for every $\widetilde{\sigma} \in \Psi^{-1}[(e, h, f)]$, there exists a spin $\widetilde{W}$-module $S$ and a Springer representation $\sigma_{(e, \phi)}$, such that $\widetilde{\sigma}$ is contained in $\sigma_{(e, \phi)} \otimes S$.

Since $\operatorname{triv}\left(\Omega_{\widetilde{W}}\right)=\operatorname{sign}\left(\Omega_{\widetilde{W}}\right)$, Theorem $2.3 .1(1)$ says in particular that any two associate genuine $\widetilde{W}$-types $\widetilde{\sigma}_{1}, \widetilde{\sigma}_{2}$ lie in the same fiber of $\Psi$.

2.4. The graded Hecke algebra. Recall the real root system $\Phi=\left(V, R, V^{\vee}, R^{\vee}\right)$. The complexifications of $V, V^{\vee}$ are denoted by $V_{\mathbb{C}}, V_{\mathbb{C}}^{\vee}$. We denote by $S\left(V_{\mathbb{C}}\right)$ the symmetric algebra in $V_{\mathbb{C}}$.

Definition 2.4.1 ( $[\mathrm{Lu}]$ ). The graded affine Hecke algebra $\mathbb{H}$ (with equal parameters) is defined as follows:

(i) as a $\mathbb{C}$-vector space, it is $S\left(V_{\mathbb{C}}\right) \otimes \mathbb{C}[W]$;

(ii) $S\left(V_{\mathbb{C}}\right)$ and $\mathbb{C}[W]$ have the usual algebra structures as subalgebras;

(iii) the cross relations are

$$
s_{\alpha} \cdot \xi-s_{\alpha}(\xi) \cdot s_{\alpha}=\langle\alpha, \xi\rangle,
$$

for every $\alpha \in \Pi$ and $\xi \in V_{\mathbb{C}}$.

Definition 2.4.2. Let $\left\{\omega_{i}: i=1, n\right\}$ and $\left\{\omega^{i}: i=1, n\right\}$ be dual bases of $V$ with respect to $\langle$,$\rangle . Define the Casimir element of \mathbb{H}: \Omega=\sum_{i=1}^{n} \omega_{i} \omega^{i} \in \mathbb{H}$.

It is easy to see that the element $\Omega$ is independent of the choice of bases and central in $\mathbb{H}$. Moreover, if $(\pi, X)$ is an irreducible $\mathbb{H}$-module with central character $\chi_{\nu}$ for $\nu \in V_{\mathbb{C}}^{\vee}$, then $\pi$ acts on $\Omega$ by the scalar $\langle\nu, \nu\rangle$.

The algebra $\mathbb{H}$ has a natural conjugate linear anti-involution defined on generators as follows:

$$
\begin{aligned}
& w^{*}=w^{-1}, \quad w \in W, \\
& \omega^{*}=-\omega+\sum_{\beta>0}(\beta, \omega) s_{\beta}, \quad \omega \in V .
\end{aligned}
$$


An $\mathbb{H}$-module $(\pi, X)$ is said to be Hermitian if there exists a Hermitian form $(,)_{X}$ on $X$ which is invariant in the sense that $(\pi(h) x, y)_{X}=\left(x, \pi\left(h^{*}\right) y\right)_{X}$, for all $h \in \mathbb{H}, x, y \in X$. If such a form exists which is also positive definite, then $X$ is said to be unitary.

For every $\omega \in V$, define

$$
\widetilde{\omega}=\omega-\frac{1}{2} \sum_{\beta>0}(\beta, \omega) s_{\beta} \in \mathbb{H} .
$$

It is immediate that $\widetilde{\omega}^{*}=-\widetilde{\omega}$.

Definition 2.4.3 ([BCT] $)$. Let $\left\{\omega_{i}\right\},\left\{\omega^{i}\right\}$ be dual bases of $V$. The Dirac element is defined as

$$
\mathcal{D}=\sum_{i} \widetilde{\omega}_{i} \otimes \omega^{i} \in \mathbb{H} \otimes C(V) .
$$

It is elementary to verify that $\mathcal{D}$ does not depend on the choice of dual bases.

We will usually work with a fixed spin module $(\gamma, S)$ for $C(V)$ and a fixed $\mathbb{H}$ module $(\pi, X)$. Define the Dirac operator for $X$ (and $S$ ) as $D=(\pi \otimes \gamma)(\mathcal{D})$.

Suppose $X$ is a Hermitian $\mathbb{H}$-module with invariant form $(,)_{X}$. Endow $X \otimes S$ with the Hermitian form $\left(x \otimes s, x^{\prime} \otimes s^{\prime}\right)_{X \otimes S}=\left(x, x^{\prime}\right)_{X}\left\langle s, s^{\prime}\right\rangle_{S}$. Analogous to results of Parthasarathy in the real case, the operator $D$ is self adjoint with respect to $(,)_{X \otimes S}$,

$$
\left(D(x \otimes s), x^{\prime} \otimes s^{\prime}\right)_{X \otimes S}=\left(x \otimes s, D\left(x^{\prime} \otimes s^{\prime}\right)\right)_{X \otimes S}
$$

Thus a Hermitian $\mathbb{H}$-module is unitary only if

$$
\left(D^{2}(x \otimes s), x \otimes s\right)_{X \otimes S} \geq 0, \quad \text { for all } x \otimes s \in X \otimes S .
$$

We write $\Delta_{\widetilde{W}}$ for the diagonal embedding of $\mathbb{C}[\widetilde{W}]$ into $\mathbb{H} \otimes C(V)$ defined by extending $\Delta_{\widetilde{W}}(\widetilde{w})=p(\widetilde{w}) \otimes \widetilde{w}$ linearly.

For $\widetilde{w} \in \widetilde{W}$, one can easily see that

$$
\Delta_{\widetilde{W}}(\widetilde{w}) \mathcal{D}=\operatorname{sign}(\widetilde{w}) \mathcal{D} \Delta_{\widetilde{W}}(\widetilde{w})
$$

as elements of $\mathbb{H} \otimes C(V)$. In particular, the kernel of the Dirac operator on $X \otimes S$ is invariant under $\widetilde{W}$.

Theorem 2.4.1 ([BCT]). The square of the Dirac element equals

$$
\mathcal{D}^{2}=-\Omega \otimes 1+\frac{1}{4} \Delta_{\widetilde{W}}\left(\Omega_{\widetilde{W}}\right),
$$

in $\mathbb{H} \otimes C(V)$.

2.5. Dirac cohomology. To have a uniform notation, we will denote a spin module by $S^{\epsilon}$. If $\operatorname{dim} V$ is even, then $S^{\epsilon}$ is $S$, the unique spin module, and if $\operatorname{dim} V$ is odd, then $\epsilon$ could be + or - .

Definition 2.5.1. In the setting of Definition 2.4.3, define

$$
H_{\epsilon}^{D}(X):=\operatorname{ker} D /(\operatorname{ker} D \cap \operatorname{Im} D)
$$

and call it the Dirac cohomology of $X$. (The symbol $\epsilon$ denotes the dependence on the spin module $S^{\epsilon}$.) If $X$ is unitary, the self-adjointness of $D$ implies that $\operatorname{ker}(D) \cap \operatorname{Im}(D)=0$, and so $H_{\epsilon}^{D}(X)=\operatorname{ker}(D)$.

Vogan's conjecture takes the following form. 
Theorem 2.5.1 ([BCT, Theorem 4.8]). Suppose $(\pi, X)$ is an $\mathbb{H}$ module with central character $\chi_{\nu}$ with $\nu \in V_{\mathbb{C}}^{\vee}$. Suppose that $H_{\epsilon}^{D}(X) \neq 0$ and let $(\widetilde{\sigma}, \widetilde{U})$ be an irreducible representation of $\widetilde{W}$ such that $\operatorname{Hom}_{\widetilde{W}}\left(\widetilde{U}, H_{\epsilon}^{D}(X)\right) \neq 0$. If $\Psi(\widetilde{\sigma})=[(e, h, f)] \in$ $\mathcal{T}_{0}(G)$, then $\nu=\frac{1}{2} h$.

As a corollary, we find the following formula for $H_{\epsilon}^{D}(X)$.

Corollary 2.5.1. Assume $X$ is an $\mathbb{H}$ module with central character $\chi_{\frac{1}{2} h}$, for some $[(e, h, f)] \in \mathcal{T}_{0}(G)$ (otherwise $H_{\epsilon}^{D}(X)=0$ ). Then, as a $\widetilde{W}$-module

$$
H_{\epsilon}^{D}(X)=\sum_{\widetilde{\sigma} \in \Psi^{-1}(e, h, f)} \sum_{\mu \in \widehat{W}}\left[\widetilde{\sigma}: \mu \otimes S^{\epsilon}\right]\left[\left.X\right|_{W}: \mu\right] \widetilde{\sigma} .
$$

Theorem 2.5.1 has an easy weak converse, which will be useful in applications.

Proposition 2.5.1. Assume that $(\pi, X)$ is a unitary $\mathbb{H}$ module with central character $\chi_{\nu}, \nu \in V_{\mathbb{C}}^{\vee}$ and that there exists an irreducible $\widetilde{W}$-type $(\widetilde{\sigma}, \widetilde{U})$ such that $\operatorname{Hom}_{\widetilde{W}}\left(\widetilde{U}, X \otimes S^{\epsilon}\right) \neq 0$ and $\langle\nu, \nu\rangle=\widetilde{\sigma}\left(\Omega_{\widetilde{W}}\right)$. Then $\operatorname{Hom}_{\widetilde{W}}\left(\widetilde{U}, H_{\epsilon}^{D}(X)\right) \neq 0$, and in particular $H_{\epsilon}^{D}(X) \neq 0$.

Proof. Let $x \otimes s$ be an element of $X \otimes S^{\epsilon}$ in the isotypic component of $\widetilde{\sigma}$. Then $D^{2}(x \otimes s)=-\langle\nu, \nu\rangle+\widetilde{\sigma}\left(\Omega_{\widetilde{W}}\right)=0$. Since $X$ is assumed unitary, the operator $D$ is selfadjoint on $X \otimes S$ and thus ker $D^{2}=\operatorname{ker} D$. This implies $x \otimes s \in \operatorname{ker} D\left(=H_{\epsilon}^{D}(X)\right.$.

2.6. An induction lemma. Let $\left(V_{M}, R_{M}, V_{M}^{\vee}, R_{M}^{\vee}\right)$ be a root subsystem of $\left(V, R, V^{\vee}, R^{\vee}\right)$. Let $\Pi_{M} \subset \Pi$ be the corresponding simple roots and $W_{M} \subset W$ the reflection subgroup. Let $\mathbb{H}_{M}$ denote the Hecke subalgebra of $\mathbb{H}$ given by this root subsystem. Denote by $V_{N}$ the orthogonal complement of $V_{M}$ in $V$ with respect to the fixed product $\langle$,$\rangle .$

Recall that the graded tensor product $A \hat{\otimes} B$ of two $\mathbb{Z} / 2 \mathbb{Z}$-graded algebras $A$ and $B$ is $A \otimes B$ as a vector space, but with multiplication defined by

$$
\left(a_{1} \otimes b_{1}\right)\left(a_{2} \otimes b_{2}\right)=(-1)^{\operatorname{deg}\left(b_{1}\right) \operatorname{deg}\left(a_{2}\right)} a_{1} a_{2} \otimes b_{1} b_{2} .
$$

Lemma 2.6.1. There is an isomorphism of algebras $C(V) \cong C\left(V_{M}\right) \hat{\otimes} C\left(V_{N}\right)$.

Proof. If an orthonormal basis of $V_{M}$ is $\left\{\omega_{1}, \ldots, \omega_{k}\right\}$ and an orthonormal basis of $V_{n}$ is $\left\{\omega_{k+1}, \ldots, \omega_{n}\right\}$, then the isomorphism is given by $\omega_{i_{1}} \ldots \omega_{i_{l}} \otimes \omega_{j_{1}} \ldots \omega_{j_{r}} \mapsto$ $\omega_{i_{1}} \ldots \omega_{i_{l}} \omega_{j_{1}} \ldots \omega_{j_{r}}$, where $i_{1}, \ldots, i_{l} \in\{1, \ldots, k\}$ and $j_{1}, \ldots, j_{r} \in\{k+1, \ldots, n\}$.

Since $W_{M}$ acts trivially on $V_{N}$, and therefore $\widetilde{W}_{M}$ acts trivially on every $C\left(V_{N}\right)$ module, we see that as $\widetilde{W}_{M}$-representations:

$S \cong \oplus_{2^{\operatorname{dim} V_{N} / 2}} S_{M}, \quad$ if $\operatorname{dim} V, \operatorname{dim} V_{M}$ are both even;

$S^{ \pm} \cong \oplus_{2 \operatorname{dim} V_{N} / 2} S_{M}^{ \pm}, \quad$ if $\operatorname{dim} V, \operatorname{dim} V_{M}$ are both odd;

$S^{ \pm} \cong \oplus_{2^{\left(\operatorname{dim} V_{N}-1\right) / 2}} S_{M}, \quad$ if $\operatorname{dim} V$ is odd and $\operatorname{dim} V_{M}$ is even;

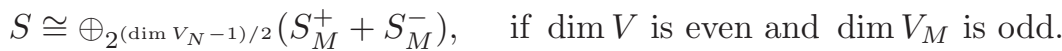

The following lemma will be our main criterion for proving that certain induced modules have nonzero Dirac cohomology. In order to reduce the number of cases, denote $\mathcal{S}=S$ if $\operatorname{dim} V$ is even, and $\mathcal{S}=S^{+}+S^{-}$if $\operatorname{dim} V$ is odd, and similarly define $\mathcal{S}_{M}$. In particular, $\mathcal{S}$ is self-contragredient.

Lemma 2.6.2. Let $\pi_{M}$ be an $\mathbb{H}_{M}$-module, and $\pi=\mathbb{H} \otimes_{\mathbb{H}_{M}} \pi_{M}$. 
(a) $\operatorname{Hom}_{\widetilde{W}}[\widetilde{\sigma}, \pi \otimes \mathcal{S}]=\frac{\operatorname{dim} \mathcal{S}}{\operatorname{dim} \mathcal{S}_{M}} \operatorname{Hom}_{\widetilde{W}_{M}}\left[\left.\widetilde{\sigma}\right|_{\widetilde{W}_{M}}, \pi_{M} \otimes \mathcal{S}_{M}\right]$.

(b) Assume that $\pi_{M}$ is unitary and the $\widetilde{W}_{M}$-type $\widetilde{\sigma}_{M}$ occurs in the $H^{D}\left(\pi_{M}\right)$. If there exists a $\widetilde{W}$-type $\widetilde{\sigma}$ such that

(i) $\operatorname{Hom}_{\widetilde{W}_{M}}\left[\widetilde{\sigma}_{M}, \widetilde{\sigma}\right] \neq 0$;

(ii) if $\Psi(\widetilde{\sigma})=[(e, h, f)]$, then the central character of $\pi$ is $\chi_{\pi}=h$, then $\widetilde{\sigma}$ occurs in $H^{D}(\pi)$.

Proof. Part (b) is an immediate consequence of (a) using Proposition 2.5.1. To prove (a), we use Frobenius reciprocity and the restriction of $\mathcal{S}$ to $\widetilde{W}_{M}$ :

$$
\begin{aligned}
\operatorname{Hom}_{\widetilde{W}}[\widetilde{\sigma}, \pi \otimes \mathcal{S}] & =\operatorname{Hom}_{W}\left[\widetilde{\sigma} \otimes \mathcal{S}, \operatorname{Ind}_{W_{M}}^{W} \pi_{M}\right]=\operatorname{Hom}_{W_{M}}\left[\left.(\widetilde{\sigma} \otimes \mathcal{S})\right|_{W_{M}}, \pi_{M}\right] \\
& =\operatorname{Hom}_{\widetilde{W}_{M}}\left[\left.\widetilde{\sigma}\right|_{\widetilde{W}_{M}},\left.\pi_{M} \otimes \mathcal{S}\right|_{\widetilde{W}_{M}}\right]=\frac{\operatorname{dim} \mathcal{S}}{\operatorname{dim} \mathcal{S}_{M}} \operatorname{Hom}_{\widetilde{W}_{M}}\left[\left.\widetilde{\sigma}\right|_{\widetilde{W}_{M}}, \pi_{M} \otimes \mathcal{S}_{M}\right] .
\end{aligned}
$$

2.7. Spherical modules. An $\mathbb{H}$-module $X$ is called spherical if $\mathrm{Hom}_{W}[\operatorname{triv}, X] \neq 0$. The (spherical) principal series modules of $\mathbb{H}$ are defined as the induced modules

$$
X(\nu)=\mathbb{H} \otimes_{S\left(V_{\mathbb{C}}\right)} \mathbb{C}_{\nu},
$$

for $\nu \in V_{\mathbb{C}}^{\vee}$. Since $X(\nu) \cong \mathbb{C}[W]$ as $W$-modules, there is a unique irreducible spherical $\mathbb{H}$-subquotient $L(\nu)$ of $X(\nu)$. It is well known that:

(1) $L(\nu) \cong L(w \nu)$, for every $w \in W$;

(2) if $\nu$ is $R^{+}$-dominant, then $L(\nu)$ is the unique irreducible quotient of $X(\nu)$;

(3) every irreducible spherical $\mathbb{H}$-module is isomorphic to a quotient $L(\nu), \nu$ $R^{+}$-dominant.

Recall the Lie algebra $\mathfrak{g}$ that we attached to the root system $\Phi$. The identification $\mathfrak{h}=V_{\mathbb{C}}^{\vee}$ allows us to view $\nu$ as an element of $\mathfrak{h}$. Next, consider $\mathfrak{g}_{1}=\{x \in \mathfrak{g}:[\nu, x]=$ $x$, the ad 1-eigenspace of $\nu$ on $\mathfrak{g}$. The stabilizer $G_{0}=\{g \in G: \operatorname{Ad}(g) \nu=\nu\}$ acts on $\mathfrak{g}_{1}$ with finitely many orbits, and let $e$ be an element of the unique open dense $G_{0}$-orbit. Lusztig's geometric realization of $\mathbb{H}$ and classification of irreducible $\mathbb{H}$-modules implies in particular the following statement.

Proposition 2.7.1. Let $\nu \in V_{\mathbb{C}}^{\vee}$ be given and let $e$ be a nilpotent element of $\mathfrak{g}$ attached to $\nu$ by the procedure above. Then the spherical module $L(\nu)$ contains the Springer representation $\sigma_{(e, 1)}$ with multiplicity one.

The second result that we need is the unitarizability of the spherical unipotent $\mathbb{H}$-modules.

Proposition 2.7.2 ( $\mathrm{BM}])$. For every Lie triple $(e, h, f)$, the spherical module $L\left(\frac{1}{2} h\right)$ is unitary.

Now we can state the classification of spherical modules with nonzero Dirac cohomology.

Definition 2.7.1. We say that an $\mathbb{H}$-module $X$ has nonzero Dirac cohomology if for a choice of spin module $S^{\epsilon}, H_{\epsilon}^{D}(X) \neq 0$.

Let $[(e, h, f)] \in \mathcal{T}_{0}(G)$ be given and assume $G$ is simple. The results of [Ci] give a concrete description in every Lie type of the map $\Psi$ from Theorem 2.3.1. In particular, there is either only one self-associate $\widetilde{W}$-type which we denote $\widetilde{\sigma}_{(e, 1)}$, or 
two associate $\widetilde{W}$-types denoted $\widetilde{\sigma}_{(e, 1)}^{ \pm}$, which appear in the fiber $\Psi^{-1}(e, h, f)$ and can occur in the decomposition of the tensor product $\sigma_{(e, 1)} \otimes S^{\epsilon}$.

Corollary 2.7.1. An irreducible spherical module $L(\nu)$ has nonzero Dirac cohomology if and only if $\nu=w \cdot \frac{1}{2} h$ for some $[(e, h, f)] \in \mathcal{T}_{0}(G)$.

Proof. Assume that $H_{\epsilon}^{D}(L(\nu)) \neq 0$. Then there exists a genuine $\widetilde{W}$-type $\widetilde{\sigma}$ occuring in $H_{\epsilon}^{D}(L(\nu))$, such that $\Psi(\widetilde{\sigma})=[(e, h, f)] \in \mathcal{T}_{0}(G)$. By Theorem 2.5.1 $\nu=w \cdot \frac{1}{2} h$.

Conversely, fix $[(e, h, f)] \in \mathcal{T}_{0}(G)$. The spherical module $L\left(\frac{1}{2} h\right)$ contains $\sigma_{(e, 1)}$ with multiplicity one by Proposition 2.7.1, and it is unitary by Proposition 2.7.2 From this, Proposition 2.5.1implies immediately that one of the $\widetilde{W}$-types in $\Psi^{-1}(e, h, f)$ occurs in $H_{\epsilon}^{D}\left(L\left(\frac{1}{2} h\right)\right)$, for some $\epsilon$, and therefore $L\left(\frac{1}{2} h\right)$ has nonzero Dirac cohomology.

In order to investigate the precise formula for $H_{\epsilon}^{D}(L(h / 2))$, one uses (2.5.2) and the results of Borho-McPherson BMcP about the $W$-structure of the Springer representations in $A(e)$-isotypic components of the full cohomology of a Springer fiber. In our setting, this says that, as a $W$-module:

$$
L(h / 2)=\sigma_{(e, 1)}+\sum_{e^{\prime}>e} \sum_{\phi^{\prime} \in \widehat{A}(e)_{0}} m_{e^{\prime}, \phi^{\prime}} \sigma_{\left(e^{\prime}, \phi^{\prime}\right)},
$$

for some integers $m_{e^{\prime}, \phi^{\prime}} \geq 0$. Here $e^{\prime}>e$ means the closure ordering of nilpotent orbits, i.e., $e \in \overline{G \cdot e^{\prime}} \backslash G \cdot e^{\prime}$. We make the following conjecture.

Conjecture 2.7.1. Let $\widetilde{\sigma}$ be a $\widetilde{W}$-type such that $\Psi(\widetilde{\sigma})=\left[\left(e^{\prime}, h^{\prime}, f^{\prime}\right)\right]$. Then

$$
\operatorname{Hom}_{\widetilde{W}}\left[\widetilde{\sigma}, \sigma_{(e, \phi)} \otimes S^{\epsilon}\right] \neq 0
$$

only if $e^{\prime} \geq e$.

If this conjecture is true, then if we tensor by $S^{\epsilon}$ in (2.7.1), every $\widetilde{W}$-type coming from a $\sigma_{\left(e^{\prime}, \phi^{\prime}\right)} \otimes S^{\epsilon}, e^{\prime}>e$, would correspond under the map $\Psi$ to a triple $\left(e^{\prime \prime}, h^{\prime \prime}, f^{\prime \prime}\right)$ with $e^{\prime \prime} \geq e^{\prime}>e$. In particular, $\left|h^{\prime \prime}\right|>|h|$, so the formula for $D_{\epsilon}^{2}$ (Theorem 2.4.1) implies that none of these $\widetilde{W}$-types can contribute to $H_{\epsilon}^{D}(L(h / 2))$. Thus the only nontrivial contribution to $H_{\epsilon}^{D}(L(h / 2))$ comes from $\sigma_{(e, 1)} \otimes S^{\epsilon}$, and we would have:

$$
\begin{aligned}
& H_{\epsilon}^{D}(L(h / 2))=\left[\widetilde{\sigma}_{(e, 1)}: \sigma_{(e, 1)} \otimes S^{\epsilon}\right] \widetilde{\sigma}_{(e, 1)}, \text { if } \widetilde{\sigma}_{(e, 1)} \cong \widetilde{\sigma}_{(e, 1)} \otimes \text { sign; } \\
& H_{\epsilon}^{D}(L(h / 2))=\left[\widetilde{\sigma}_{(e, 1)}^{+}: \sigma_{(e, 1)} \otimes S^{\epsilon}\right] \widetilde{\sigma}_{(e, 1)}^{+}+\left[\widetilde{\sigma}_{(e, 1)}^{-}: \sigma_{(e, 1)} \otimes S^{\epsilon}\right] \widetilde{\sigma}_{(e, 1)}^{-}, \text {otherwise. }
\end{aligned}
$$

In section 3 we will show that Conjecture 2.7 .1 holds when $\mathbb{H}$ is a Hecke algebra of type $A$, and therefore in that case (2.7.2) is true (see Lemma 3.6.2). Further evidence for this conjecture is provided by the computation of the Dirac index for tempered $\mathbb{H}$-modules in $\mathrm{CT}$, Theorem 1].

\section{Nonzero Dirac cohomology for type $A$}

In this section, we specialize to the case of the graded Hecke algebra attached to the root system $\Phi=\left(V, R, V^{\vee}, R^{\vee}\right)$ of $g l(n)$. Explicitly, $V=\mathbb{R}^{n}$ with a basis $\left\{\epsilon_{1}, \ldots, \epsilon_{n}\right\}, R=\left\{\epsilon_{i}-\epsilon_{j}: 1 \leq i \neq j \leq n\right\}$. To simplify notation, we will also use the coordinates $\left\{\epsilon_{i}\right\}$ to describe $V^{\vee} \cong \mathbb{R}^{n}$ and $R^{\vee}$. We choose positive roots $R^{+}=$ $\left\{\epsilon_{i}-\epsilon_{j}: 1 \leq i<j \leq n\right\}$. The simple roots are therefore $\Pi=\left\{\epsilon_{i}-\epsilon_{i+1}: 1 \leq i<n\right\}$. 
The Weyl group is the symmetric group $S_{n}$ and we write $s_{i, j}$ for the reflection in the root $\epsilon_{i}-\epsilon_{j}$.

The graded Hecke algebra $\mathbb{H}_{n}$ for $g l(n)$ is therefore generated by $S_{n}$ and the set $\left\{\epsilon_{i}: 1 \leq i \leq n\right\}$ subject to the commutation relations:

$$
\begin{aligned}
& s_{i, i+1} \epsilon_{k}=\epsilon_{k} s_{i, i+1}, \quad k \neq i, i+1 ; \\
& s_{i, i+1} \epsilon_{i}-\epsilon_{i+1} s_{i, i+1}=1 .
\end{aligned}
$$

We review the classification of the unitary dual of $\mathbb{H}_{n}$ and then determine which unitary $\mathbb{H}_{n}$-modules have nonzero Dirac cohomology.

3.1. Langlands classification. We begin by recalling the Langlands classification for $\mathbb{H}_{n}$.

Definition 3.1.1. The Steinberg module $\mathrm{St}$ is the $\mathbb{H}_{n}$-module whose restriction to $\mathbb{C}\left[S_{n}\right]$ is the sign-representation, and whose only $S\left(V_{\mathbb{C}}\right)$ weight is $-\rho^{\vee}=-\frac{1}{2} \sum_{\alpha \in R^{+}} \alpha^{\vee}$.

Let $\lambda=\left(n_{1}, n_{2}, \ldots, n_{r}\right)$ be a composition of $n$. (This means that $n_{1}+n_{2}+\cdots+$ $n_{r}=n$, but there is no order assumed between the $n_{i}$ 's, e.g. $(2,1)$ and $(1,2)$ are different compositions of 3.) For every $1 \leq i \leq r$, regard the Hecke algebra $\mathbb{H}_{n_{i}}$ as the subalgebra of $\mathbb{H}$ generated by $\left\{\epsilon_{j}, \epsilon_{j+1}, \ldots, \epsilon_{j+n_{i}-1}\right\}$ and $\left\{s_{j, j+1}, s_{j+1, j+2}, \ldots, s_{j+n_{i}-1, j+n_{i}}\right.$, where $j=n_{1}+n_{2}+\cdots+n_{i-1}+1$. Then

$$
\mathbb{H}_{\lambda}=\mathbb{H}_{n_{1}} \times \mathbb{H}_{n_{2}} \times \cdots \times \mathbb{H}_{n_{r}}
$$

is a (parabolic) subalgebra of $\mathbb{H}_{n}$. For every $r$-tuple $\underline{\nu}=\left(\nu_{1}, \nu_{2}, \ldots, \nu_{r}\right)$ of complex numbers, we may consider the induced module

$$
I_{\lambda}(\underline{\nu})=\mathbb{H}_{n} \otimes_{\mathbb{H}_{\lambda}}\left(\mathrm{St} \otimes \mathbb{C}_{\nu_{1}}\right) \otimes \cdots \otimes\left(\mathrm{St} \otimes \mathbb{C}_{\nu_{r}}\right) .
$$

If $\underline{\nu}$ satifies the dominance condition

$$
\operatorname{Re}\left(\nu_{1}\right) \geq \operatorname{Re}\left(\nu_{2}\right) \geq \cdots \geq \operatorname{Re}\left(\nu_{r}\right),
$$

we call $I_{\lambda}(\underline{\nu})$ a standard module.

Theorem 3.1.1 $(\underline{\mathrm{BZ}})$. $\quad$ (a) Let $\lambda$ be a composition of $n$ and $I_{\lambda}(\underline{\nu})$ a standard module as in (3.1.1) and (3.1.2). Then $I_{\lambda}(\underline{\nu})$ has a unique irreducible quotient $L_{\lambda}(\underline{\nu})$.

(b) Every irreducible $\mathbb{H}_{n}$-module is isomorphic to an $L_{\lambda}(\underline{\nu})$ as in (a).

Recall that by Young's construction, the $S_{n}$-types are in one to one correspondence with partitions of $n$. We write $\sigma_{\lambda}$ for the $S_{n}$-type parameterized by the partition $\lambda$ of $n$. For example, $\sigma_{(n)}=$ triv and $\sigma_{\left(1^{n}\right)}=$ sign. If $\lambda^{t}$ denotes the transpose partition of $\lambda$, then $\sigma_{\lambda} \otimes \operatorname{sign}=\sigma_{\lambda^{t}}$. Finally, every composition $\lambda$ of $n$ gives rise to a partition of $n$ by reordering, and we denote the corresponding $S_{n}$-type by $\sigma_{\lambda}$ again.

Theorem 3.1.2 ([이 $)$. In the notation of Theorem [3.1.1, the irreducible module $L_{\lambda}(\underline{\nu})$ contains the $S_{n}$-type $\sigma_{\lambda^{t}}$ with multiplicity one.

3.2. Speh modules. The building blocks of the unitary dual are the Speh modules whose construction we review now.

The following lemma is well-known and elementary. 
Lemma 3.2.1. For every $c \in \mathbb{C}$, there exists a surjective algebra homomorphism $\tau_{c}: \mathbb{H} \rightarrow \mathbb{C}\left[S_{n}\right]$ given by:

$$
\begin{aligned}
& w \mapsto w, \quad w \in S_{n} ; \\
& \epsilon_{k} \mapsto s_{k, k+1}+s_{k, k+2}+\cdots+s_{k, n}+c, \quad 1 \leq k<n ; \\
& \epsilon_{n} \mapsto c .
\end{aligned}
$$

Proof. We check the commutation relations. It is clear that if $k \neq i, i+1, s_{i, i+1}$ commutes with $\epsilon_{k}$, since $s_{i, i+1}$ commutes with every $s_{j, n}, k \leq j<n$, when $i+1<k$, and it commutes with $s_{k, i}+s_{k, i+1}$ and $s_{k, j}, j \neq i, i+1$, when $i>k$.

Next, $s_{i, i+1} \epsilon_{i}=1+\sum_{j>i+1} w_{(i, j, i+1)}+c s_{i, i+1}$ and $\epsilon_{i+1} s_{i, i+1}=\sum_{j>i+1} w_{(i, j, i+1)}+$ $c s_{i, i+1}$, where $w_{(i, j, i+1)}$ denotes the element of $S_{n}$ with cycle structure $(i, j, i+1)$. The claim follows.

For every partition $\lambda$ of $n$ and $c \in \mathbb{C}$, define the $\mathbb{H}$-module $\tau_{c}^{*}(\lambda)$ obtained by pulling back $\sigma_{\lambda}$ to $\mathbb{H}$ via $\tau_{c}$.

Viewing $\lambda$ as a left justified Young diagram, define the $c$-content of the $(i, j)$ box of $\lambda$ to be $c+(j-i)$, and the $c$-content of $\lambda$ to be the set of $c$-contents of boxes. This is best explained by an example. If $\lambda$ is the partition of $(3,3,1)$ of $n=7$, the 0-content is the Young tableau

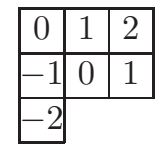

For the $c$-content, add $c$ to the entry in every box.

Lemma 3.2.2. The central character of $\tau_{c}^{*}(\lambda)$ is the $\left(S_{n}\right.$-orbit of the) c-content of the partition $\lambda$.

Proof. This follows from the known values of the simultaneous eigenvalues of the Jucys-Murphy elements $s_{k, k+1}+s_{k, k+2}+\cdots+s_{k, n}$ used to defined $\tau_{c}$. See for example [OV], Theorem 5.8].

Definition 3.2.1. If $\lambda$ is a box partition, i.e., $\lambda=(\underbrace{m, m, \ldots, m}_{d})$, for some $m, d$ such that $n=m d$, and $c=0$ when $m+d$ is even or $c=\frac{1}{2}$ when $m+d$ is odd, call the module $\tau_{c}^{*}(\lambda)$ a Speh module, and denote it by $a(m, d)$.

Lemma 3.2.3. In the notation of Theorem 3.1.1, the Speh module $a(m, d)$ is isomorphic to $L_{\lambda^{t}}\left(\frac{m-1}{2}, \frac{m-3}{2}, \ldots,-\frac{m-1}{2}\right)$, where $\lambda^{t}=(\underbrace{d, d, \ldots, d}_{m})$.

Proof. This is immediate from Theorem 3.1.1, Theorem 3.1.2 and Lemma 3.2.2.

3.3. The unitary dual. The classification of irreducible $\mathbb{H}_{n}$-modules which admit a nondegenerate invariant hermitian form is a particular case of the classical result of [KZ], as formulated in the Hecke algebra setting by [BM].

If $\lambda=\left(n_{1}, \ldots, n_{r}\right)$ is a composition of $n$, let $R_{\lambda} \subset R$ denote the root subsystem of the Levi subalgebra $g l\left(n_{1}\right) \oplus \cdots \oplus g l\left(n_{r}\right) \subset g l(n)$. If $w \in S_{n}$ has the property that $w R_{\lambda}^{+}=R_{\lambda}^{+}$, then $w$ gives rise to an algebra automorphism of $\mathbb{H}_{\lambda}$, and therefore $w$ acts on the set of irreducible $\mathbb{H}_{\lambda}$-modules. 
Theorem 3.3.1. Let $\lambda=\left(n_{1}, \ldots, n_{r}\right)$ be a composition of $n$, and $\underline{\nu}=\left(\nu_{1}, \ldots, \nu_{r}\right)$ be a dominant $r$-tuple of complex numbers in the sense of (3.1.2). In the notation of Theorem [3.1.1. $L_{\lambda}(\underline{\nu})$ is hermitian if and only if there exists $w \in S_{n}$ such that $w R_{\lambda}^{+}=R_{\lambda}^{+}$and

$$
w\left(\left(\mathrm{St} \otimes \mathbb{C}_{\nu_{1}}\right) \otimes \cdots \otimes\left(\mathrm{St} \otimes \mathbb{C}_{\nu_{r}}\right)\right)=\left(\mathrm{St} \otimes \mathbb{C}_{-\bar{\nu}_{1}}\right) \otimes \cdots \otimes\left(\mathrm{St} \otimes \mathbb{C}_{-\bar{\nu}_{r}}\right),
$$

as $\mathbb{H}_{\lambda}$-modules.

Corollary 3.3.1. Every Speh module $a(m, d)$ is a unitary $\mathbb{H}_{n}$-module.

Proof. Let $w_{0}$ denote the longest Weyl group element in $S_{n}$ and $w_{0}(\lambda)$ the longest Weyl group element in $S_{n_{1}} \times \cdots \times S_{n_{r}}$. Using Lemma 3.2.3, we see now that every Speh module $a(m, d)$ is hermitian since the Weyl group element $w_{0} w_{0}\left(\lambda^{t}\right)$ satifies condition (3.3.1) in this case.

Since in addition $a(m, d)$ is irreducible as an $S_{n}$-module, it is in fact unitary.

The classification of the unitary dual of $\mathbb{H}_{n}$ is also well-known (see Ta for the classification of the unitary dual for $G L\left(n, \mathbb{Q}_{p}\right)$ ).

The building blocks are the Speh modules defined before. First, every Speh module $a(m, d)$ can be tensored with a unitary character $\mathbb{C}_{y}, y \in \sqrt{-1} \mathbb{R}$ by which the central element $\epsilon_{1}+\cdots+\epsilon_{n}$ of $\mathbb{H}_{n}$ acts. We denote the resulting (unitary) irreducible module by $a_{y}(m, d)$.

Next, we consider induced complementary series representations of the form

$$
\pi\left(a_{y}(m, d), \nu\right)=\mathbb{H}_{2 k} \otimes_{\mathbb{H}_{k} \times \mathbb{H}_{k}}\left(a_{y}(m, d) \otimes \mathbb{C}_{\nu}\right) \otimes\left(a_{y}(m, d) \otimes \mathbb{C}_{-\nu}\right), \quad 0<\nu<\frac{1}{2} ;
$$

in this notation, it is implicit that $k=m d$. An easy deformation argument shows that all $\pi\left(a_{y}(m, d)\right)$ are irreducible unitary $\mathbb{H}_{2 k}$-modules.

Theorem 3.3.2 ([a]). $\quad$ (a) Let $\lambda=\left(n_{1}, \ldots, n_{r}\right)$ be a composition of $n$. If every $\pi_{1}, \ldots, \pi_{r}$ is either a Speh module of the form $a_{y}(m, d)$ or an induced complementary series module of the form $\pi\left(a_{y}(m, d), \nu\right)$ as in (3.3.2), then the induced module

$$
\mathbb{H} \otimes_{\mathbb{H}_{\lambda}}\left(\pi_{1} \otimes \cdots \otimes \pi_{r}\right)
$$

is irreducible and unitary. Moreover, two such modules are isomorphic if and only if one is obtained from the other one by permuting the factors.

(b) Every unitary $\mathbb{H}_{n}$-module is of the form (3.3.3).

3.4. Nilpotent orbits in $\operatorname{sl}(n)$. The classification of nilpotent orbits for $\operatorname{sl}(n)$ is well-known. Let $P(n)$ denote the set of all (decreasing) partitions of $n$ and let $D P(n)$ be the set of partitions with distinct sizes. The Jordan canonical form gives a bijection between the set of nilpotent orbits of $s l(n)$ and $P(n)$. If $\left(e_{\lambda}, h_{\lambda}, f_{\lambda}\right)$ is a Lie triple, where the nilpotent element $e_{\lambda}$ is the Jordan form given by the partition $\lambda=\left(n_{1}, n_{2}, \ldots, n_{r}\right), n_{1} \geq n_{2} \geq \cdots \geq n_{r}>0$, then, using the identification $\mathfrak{h}=\mathbb{C}^{n}$, the middle element $h_{\lambda}$ can be chosen to have coordinates

$$
h_{\lambda}=\left(\frac{n_{1}-1}{2}, \ldots,-\frac{n_{1}-1}{2} ; \ldots ; \frac{n_{r}-1}{2}, \ldots,-\frac{n_{r}-1}{2}\right) .
$$

If we write $\lambda$ as $\lambda=(\underbrace{n_{1}^{\prime}, \ldots, n_{1}^{\prime}}_{k_{1}}, \underbrace{n_{2}^{\prime}, \ldots, n_{2}^{\prime}}_{k_{2}}, \ldots, \underbrace{n_{l}^{\prime}, \ldots, n_{l}^{\prime}}_{k_{l}})$, with $n_{1}^{\prime}>n_{2}^{\prime}>\cdots>$ $n_{l}^{\prime}>0$, then the centralizer in $g l(n)$ of the triple $\left(e_{\lambda}, h_{\lambda}, f_{\lambda}\right)$ is $g l\left(k_{1}\right) \oplus g l\left(k_{2}\right) \oplus$ 
$\cdots \oplus g l\left(k_{l}\right)$. In particular, the centralizer in $\operatorname{sl}(n)$ is a toral subalgebra if and only if $\lambda \in D P(n)$. Thus, we have a natural bijection $\mathcal{T}_{0}(S L(n)) \leftrightarrow D P(n)$. For $\lambda \in P(n)$, $\left(\mathcal{T}_{0}\right.$ defined in (2.3.5) ) viewed as a left justified Young tableau, define

$$
\operatorname{hook}(\lambda)
$$

to be the partition obtained by taking the hooks of $\lambda$. For example, if $\lambda=(3,3,1)$, then $\operatorname{hook}(\lambda)=(5,2)$. It is clear that $\operatorname{hook}(\lambda) \in D P(n)$.

We will need the following reformulation for the central character of a Speh module.

Lemma 3.4.1. The central character of a Speh module $a(m, d)$ is the ( $S_{n}$-orbit of) $h_{\lambda^{\prime}}$ (see 3.4.1)), where $\lambda^{\prime}$ is the partition

$$
\lambda^{\prime}=\operatorname{hook}(\underbrace{m, m, \ldots, m}_{d})=(m+d-1, m+d-3, \ldots,|m-d|+1) .
$$

Proof. This is immediate from Lemma 3.2 .2 and (3.4.1).

3.5. Irreducible $\widetilde{S}_{n}$-representations. Denote the length of a partition $\lambda$ by $|\lambda|$. We say that $\lambda$ is even (resp. odd) if $n-|\lambda|$ is even (resp. odd). The first part of Theorem 2.3.1 for $\widetilde{S}_{n}$ is a classical result of Schur.

Theorem 3.5.1 (Schur, [St]). The irreducible $\widetilde{S}_{n}$-representations are parameterized by partitions in $D P(n)$ as follows:

(i) for every even $\lambda \in D P(n)$, there exists a unique $\widetilde{\sigma}_{\lambda} \in{\widehat{\widehat{S}_{n}}}_{n}$;

(ii) for every odd $\lambda \in D P(n)$, there exist two associate $\widetilde{\sigma}_{\lambda}^{+}, \widetilde{\sigma}_{\lambda}^{-} \in \widehat{\widetilde{S}_{n}}$.

The dimension of $\widetilde{\sigma}_{\lambda}$ or $\widetilde{\sigma}_{\lambda}^{ \pm}$, where $\lambda=\left(\lambda_{1}, \ldots, \lambda_{m}\right) \in D P(n)$, is

$$
2^{\left[\frac{n-m}{2}\right]} \frac{n !}{\lambda_{1} ! \ldots \lambda_{m} !} \prod_{1 \leq i<j \leq m} \frac{\lambda_{i}-\lambda_{j}}{\lambda_{i}+\lambda_{j}} .
$$

In order to simplify the formulas below, we let $\widetilde{\sigma}_{\lambda}^{\epsilon}$ denote any one of $\widetilde{\sigma}_{\lambda}$, if $\lambda$ is an even partition in $D P(n)$, or $\widetilde{\sigma}_{\lambda}^{ \pm}$, if $\lambda$ is an odd partition in $D P(n)$.

The decomposition of the tensor product of an $S_{n}$-type $\sigma_{\mu}$ with a spin representation $\widetilde{\sigma}_{(n)}$ is known.

Theorem 3.5.2 ([St, Theorem 9.3], [Ma, Chapter 3, (8.17)]). If $\lambda \neq(n)$, we have:

$$
\operatorname{dim} \operatorname{Hom}_{\widetilde{S}_{n}}\left[\widetilde{\sigma}_{\lambda}, \sigma_{\mu} \otimes \widetilde{\sigma}_{(n)}\right]=\frac{1}{\epsilon_{\lambda} \epsilon_{(n)}} 2^{\frac{|\lambda|-1}{2}} g_{\lambda, \mu},
$$

where $\epsilon_{\lambda}=1$ (resp. $\epsilon_{\lambda}=\sqrt{2}$ ) if $\lambda$ is even (resp. odd), and the integer $g_{\lambda, \mu}$ is the $(\lambda, \mu)$ entry in the inverse matrix $K(-1)^{-1}$, where $K(t)$ is the matrix of KostkaFoulkes polynomials. In particular:

(i) $g_{\lambda, \lambda}=1$;

(ii) $g_{\lambda, \mu}=0$, unless $\lambda \geq \mu$ in the ordering of partitions.

Example 3.5.1. The integers $g_{\lambda, \mu}$ have also an explicit combinatorial description in terms of "shifted tableaux" of unshifted shape $\mu$ and content $\lambda$ satifying certain admissibility conditions (see [St, Theorem 9.3]). From this description, one may see for example that if $\lambda=\operatorname{hook}(\mu)$, then $g_{\lambda, \mu}=1$ in (3.5.2). 
3.6. Nonzero cohomology. We are now in position to determine the unitary modules of $\mathbb{H}_{n}$ with nonzero Dirac cohomology.

We remark that since $g l(n)$ is not semisimple, the spin modules $S^{\epsilon}$ of $C(V)$ $\left(V \cong \mathbb{C}^{n}\right)$ are not necessarily irreducible $\widetilde{S}_{n}$-representations. More precisely, using (2.6.1), we see that $\left.S^{ \pm}\right|_{\widetilde{S}_{n}}=\widetilde{\sigma}_{(n)}$, when $n$ is odd, and $\left.S\right|_{\widetilde{S}_{n}}=\widetilde{\sigma}_{(n)}^{+}+\widetilde{\sigma}_{(n)}^{-}$, when $n$ is even.

Lemma 3.6.1. Assume $X$ is an irreducible $\mathbb{H}_{n}$-module such that $H^{D}(X) \neq 0$. Then the central character of $X$ is in the set $\left\{h_{\lambda} / 2: \lambda \in D P(n)\right\}$, where $h_{\lambda}$ is as in 3.4 .1 .

Proof. This is just a reformulation of Theorem 2.5.1 in this particular case.

As a consequence of (3.5.2), we obtain the following precise results for Dirac cohomology.

\section{Lemma 3.6.2.}

(a) A spherical module $L(\nu)$ has nonzero Dirac cohomology if and only if $\nu \in\left\{h_{\lambda} / 2\right.$ : $\lambda \in D P(n)\}$, where $h_{\lambda}$ is as in 3.4.1), and in this case $H_{\epsilon}^{D}\left(L\left(h_{(n)} / 2\right)\right)=S^{\epsilon}$, and if $\lambda \neq(n)$ :

$$
\begin{array}{rlrl}
H_{\epsilon}^{D}\left(L\left(h_{\lambda} / 2\right)\right) & =2^{[(|\lambda|-1) / 2]} \widetilde{\sigma}_{\lambda}, & & \text { if } n \text { is odd and } \lambda \text { is even; } \\
& =2^{[(|\lambda|-1) / 2]}\left(\widetilde{\sigma}_{\lambda}^{+}+\widetilde{\sigma}_{\lambda}^{-}\right), & \text {if } n \text { is odd and } \lambda \text { is odd } ; \\
& =2^{[(|\lambda|) / 2-1]}\left(\widetilde{\sigma}_{\lambda}^{\epsilon}+\widetilde{\sigma}_{\lambda}^{\epsilon} \otimes \operatorname{sign}\right), & \text { if } n \text { is even } .
\end{array}
$$

(b) Every Speh module a $(m, d)$ has nonzero Dirac cohomology. More precisely, $H_{\epsilon}^{D}(a(m, d))=2^{(d-1) / 2}\left(\widetilde{\sigma}_{(m+d-1, m+d-3, \ldots,|m-d|+1)}^{+}+\widetilde{\sigma}_{(m+d-1, m+d-3, \ldots,|m-d|+1)}^{-}\right)$, if $d$ is odd and $m$ is even, $H_{\epsilon}^{D}(a(m, d))=2^{[(d-1) / 2]} \widetilde{\sigma}_{(m+d-1, m+d-3, \ldots,|m-d|+1)}^{\epsilon}$, if $d$ is odd and $m$ is odd, or $H_{\epsilon}^{D}(a(m, d))=2^{[(d+1) / 2]} \widetilde{\sigma}_{(m+d-1, m+d-3, \ldots,|m-d|+1)}^{\epsilon}$, otherwise.

(c) Every complementary series induced module $\pi\left(a_{y}(m, d), \nu\right)$ as in (3.3.2) has zero Dirac cohomology.

Proof. (a) This is immediate by (2.7.1) and the upper unitriangular property of the numbers $g_{\lambda, \mu}$ in Theorem 3.5 .2

(b) By Lemma 3.4.1, the central character of $a(m, d)$ is $h_{\lambda^{\prime}}$ where $\lambda^{\prime}=\operatorname{hook}(\lambda) \in$ $D P(n)$. By Example 3.5 .1 the genuine $\widetilde{S}_{n^{-}}$type $\widetilde{\sigma}_{\lambda^{\prime}}$ occurs with nonzero multiplicity in $\sigma_{(m, m, \ldots, m)} \otimes S$. By construction, $a(m, d)$ is isomorphic with $\sigma_{(m, m, \ldots, m)}$ as $S_{n^{-}}$ representations. This means that the hypothesis of Proposition 2.5.1 are satisfied, hence $\widetilde{\sigma}_{\lambda^{\prime}}$ occurs in $H^{D}(a(m, d))$.

(c) This is immediate from Lemma 3.6.1 since $a_{y}(m, d), y \neq 0$ and $\pi\left(a_{y}(m, d), \nu\right)$, $0<\nu<\frac{1}{2}$ do not have the allowable central characters. 
Theorem 3.6.1. An irreducible unitary $\mathbb{H}_{n}$-module has nonzero Dirac cohomology if and only if it is isomorphic with an induced module

$$
\begin{aligned}
& X=\mathbb{H}_{n} \otimes_{\mathbb{H}_{e v} \otimes \mathbb{H}_{\text {odd }}}\left(\pi_{e v} \otimes \pi_{\text {odd }}\right), \quad \text { where } \\
& \mathbb{H}_{e v}=\mathbb{H}_{k_{1}} \times \mathbb{H}_{k_{2}} \times \cdots \times \mathbb{H}_{k_{\ell}}, \mathbb{H}_{\text {odd }}=\mathbb{H}_{k_{1}^{\prime}} \times \mathbb{H}_{k_{2}^{\prime}} \times \cdots \times \mathbb{H}_{k_{t}^{\prime}}, \quad \text { and } \\
& \pi_{e v}=a\left(m_{1}, d_{1}\right) \otimes a\left(m_{2}, d_{2}\right) \otimes \cdots \otimes a\left(m_{\ell}, d_{\ell}\right), \pi_{o d d}=a\left(m_{1}^{\prime}, d_{1}^{\prime}\right) \otimes a\left(m_{2}^{\prime}, d_{2}^{\prime}\right) \otimes \cdots \otimes a\left(m_{t}^{\prime}, d_{t}^{\prime}\right), \\
& m_{i}+d_{i} \equiv 0(\bmod 2), m_{j}^{\prime}+d_{j}^{\prime} \equiv 1(\bmod 2), \\
& k_{1}+k_{2}+\cdots+k_{\ell}+k_{1}^{\prime}+k_{2}^{\prime}+\cdots+k_{t}^{\prime}=n \text {, where } a\left(m_{i}, d_{i}\right), a\left(m_{j}^{\prime}, d_{j}^{\prime}\right) \text { are Speh modules } \\
& \text { for } \mathbb{H}_{k_{i}}, \mathbb{H}_{k_{j}^{\prime}} \text { and such that the following conditions are satisfied: } \\
& m_{1}+d_{1}-1 \geq\left|m_{1}-d_{1}\right|+1>m_{2}+d_{2}-1 \geq\left|m_{2}-d_{2}\right|+1>\cdots>m_{\ell}+d_{\ell}-1 ; \\
& m_{1}^{\prime}+d_{1}^{\prime}-1 \geq\left|m_{1}^{\prime}-d_{1}^{\prime}\right|+1>m_{2}^{\prime}+d_{2}^{\prime}-1 \geq\left|m_{2}^{\prime}-d_{2}^{\prime}\right|+1>\cdots>m_{t}^{\prime}+d_{t}^{\prime}-1 .
\end{aligned}
$$

Proof. From Theorem 3.3.2 a unitary irreducible module $X$ is induced from a combination of Speh modules and complementary series modules. It is immediate that in order for $X$ to have one of the central characters from Lemma 3.6.1, a first restriction is that only Speh modules can appear in the induction, so $X$ is of the form (3.6.1). Notice then that the central character of $X$ is obtained by concatenating the central characters of $a\left(m_{i}, d_{i}\right)$. Therefore the central character of $X$ is $S_{n}$-conjugate to $h_{\lambda}$, where $\lambda$ is the composition $\lambda=\lambda^{1} \sqcup \cdots \sqcup \lambda^{\ell} \sqcup \mu^{1} \sqcup \cdots \sqcup \mu^{t}$, where $\lambda^{i}=\left(m_{i}+d_{i}-1, m_{i}+d_{i}-3, \ldots,\left|m_{i}-d_{i}\right|+1\right), 1 \leq i \leq \ell$ and $\mu^{j}=$ $\left(m_{j}^{\prime}+d_{j}^{\prime}-1, m_{j}^{\prime}+d_{j}^{\prime}-3, \ldots,\left|m_{j}^{\prime}-d_{j}^{\prime}\right|+1\right), 1 \leq j \leq t$. The entries in the first type of strings are all even, while the entries in the second type of strings are all odd. Since we need $\lambda$ to have no repetitions, condition (3.6.2) follows.

For the converse, assume $X$ is as in (3.6.1) and (3.6.2). Then the central character of $X$ is $h_{\lambda}$, where $\lambda$ is as above. By Proposition 2.5.1, it remains to check that $X \otimes S$ contains the $\widetilde{S}_{n}$-type $\widetilde{\sigma}_{\lambda}$. From Lemma 2.6.2, we see that $\operatorname{Hom}_{\widetilde{S}_{n}}\left[\widetilde{\sigma}_{\lambda}, X \otimes S\right]=$ $\frac{\operatorname{dim} \mathcal{S}}{\operatorname{dim} \mathcal{S}_{M}} \operatorname{Hom}_{\widetilde{W}_{M}}\left[\left.\widetilde{\sigma}\right|_{\widetilde{W}_{M}},\left.\left(\pi_{e v} \otimes \pi_{o d d}\right)\right|_{W_{M}} \otimes \mathcal{S}_{M}\right]$, where $\widetilde{W}_{M}=\widetilde{S}_{k_{1}} \ldots . \widetilde{S}_{k_{\ell}} \cdot \widetilde{S}_{k_{1}^{\prime}} \ldots . \widetilde{S}_{k_{t}^{\prime}}$, and $\mathcal{S}_{M}$ is the corresponding spin module. (Here $\cdot$ denotes the graded version of the direct product coming from the graded tensor product of Clifford algebras as in Section 2.6.) From Lemma 3.6.2, we know that the $\widetilde{S}_{k_{i}}$-representation $\widetilde{\sigma}_{\lambda^{i}}$ occurs in $\left.a\left(m_{i}, d_{i}\right)\right|_{S_{k_{i}}}$ tensored with the spin $\widetilde{S}_{k_{i}}$-module and similarly the $\widetilde{S}_{k_{j}^{\prime}}$-representation $\widetilde{\sigma}_{\mu^{j}}$ occurs in $\left.a\left(m_{j}^{\prime}, d_{j}^{\prime}\right)\right|_{S_{k_{j}^{\prime}}}$ tensored with the spin $\widetilde{S}_{k_{j}^{\prime}}$-module. Therefore the tensor product representation $\widetilde{\sigma}_{\lambda, M}^{j}:=\widetilde{\sigma}_{\lambda^{1}} \otimes \cdots \otimes \widetilde{\sigma}_{\lambda^{\ell}} \otimes \widetilde{\sigma}_{\mu^{1}} \otimes \cdots \otimes \widetilde{\sigma}_{\mu^{t}}$ occurs in $\left(\pi_{e v} \otimes\right.$ $\left.\pi_{o d d}\right)\left.\right|_{W_{M}} \otimes \mathcal{S}_{M}$. Finally, since the composition $\lambda$ is just the concatenation of the $\left(\lambda^{i}\right)$ 's and the $\left(\mu^{j}\right)$ 's, one sees that $\widetilde{\sigma}_{\lambda, M}$ occurs with nonzero multiplicity in $\left.\widetilde{\sigma}_{\lambda}\right|_{\widetilde{W}_{M}}$.

\section{REFERENCES}

[A] J. Arthur, Unipotent automorphic representations: conjectures. Orbites unipotentes et représentations, II, Astérisque No. 171-172 (1989), 13-71.

[AS] M. Atiyah, W. Schmid, A geometric construction of the discrete series for semisimple Lie groups, Invent. Math. 42 (1977), 1-62.

[BCT] D. Barbasch, D. Ciubotaru, P. Trapa, The Dirac operator for graded affine Hecke algebras, to appear in Acta Math.

[BM] D. Barbasch, A. Moy, Unitary spherical spectrum for p-adic classical groups, Acta Appl. Math. 44 (1996), no. 1-2, 3-37. 
[BMcP] W. Borho, R. MacPherson Partial resolutions of nilpotent varieties, Analysis and topology on singular spaces, II, III (Luminy, 1981), 23-74, Astérisque, 101-102, Soc. Math. France, Paris, 1983.

[BZ] J. Bernstein, A. Zelevinsky, Induced representations of reductive p-adic groups. I, Ann. Sci. École Norm. Sup. (4) 10 (1977), no. 4, 441-472.

[BW] A. Borel, N. Wallach, Continuous cohomology, discrete subgroups, and representations of reductive groups, Princeton University Press, Princeton, New Jersey, 1980.

[Ch] C. Chevalley, The algebraic theory of spinors, Columbia University Press, New York, 1954. viii+131 pp.

[Ci] D. Ciubotaru, Spin representations of Weyl groups and Springer's correspondence, to appear in J. Reine Angew. Math.

[CT] D. Ciubotaru, P. Trapa, Characters of Springer representations on elliptic conjugacy classes, to appear in Duke Math.

[EW] T. Enright, N. Wallach Embeddings of unitary highest weight representations and generalized Dirac operators, Math. Ann. 307 (1997), no. 4, 627-646.

[HP] J.-S. Huang, P. Pandzic̀, Dirac cohomology, unitary representations and a proof of a conjecture of Vogan, J. Amer. Math. Soc. 15 (2002), no. 1, 185-202.

[KZ] A. Knapp, G. Zuckerman, Classification theorems for representations of semisimple Lie groups, Non-commutative harmonic analysis (Actes Colloq., Marseille-Luminy, 1976), 138159. Lecture Notes in Math., Vol. 587, Springer, Berlin, 1977.

[Lu] G. Lusztig, Affine Hecke algebras and their graded version, J. Amer. Math. Soc. 2 (1989), 599-635.

[Ma] I.G. MacDonald, Symmetric functions and Hall polynomials, Oxford Mathematical Monographs, Oxford Science Publications, The Clarendon Press, Oxford University Press, New York, 1995. $\mathrm{x}+475 \mathrm{pp}$.

[Mo] A. Morris, Projective representations of reflection groups. II, Proc. London Math. Soc. (3) 40 (1980), no. 3, 553-576.

[OV] A. Okounkov, A. Vershik, A new approach to representation theory of symmetric groups, Selecta Math.2 (4) (1996), 581-605.

[P] R. Parthasarathy, Dirac operator and the discrete series, Ann. of Math. (2) 96 (1972), 1-30.

[Ro] J. Rogawski, On modules over the Hecke algebra of a p-adic group, Invent. Math. 79 (1985), no. 3, 443-465.

[St] J. Stembridge, Shifted tableaux and the projective representations of symmetric groups, Adv. Math. 74 (1989), no. 1, 87-134.

[Ta] M. Tadić, Classification of unitary representations in irreducible representations of general linear group (non-Archimedean case), Ann. Sci. École Norm. Sup. (4) 19 (1986), no. 3, 335382.

(D. Barbasch) Dept. of Mathematics, Cornell University, Ithaca, NY 14850

E-mail address: barbasch@math.cornell.edu

(D. Ciubotaru) Dept. of Mathematics, University of Utah, Salt Lake City, Ut 84112

E-mail address: ciubo@math.utah.edu 